\title{
A IMPORTÂNCIA DA QUÍMICA ANALÍTICA QUALITATIVA NOS CURSOS DE QUÍMICA DAS INSTITUIÇÕES DE ENSINO SUPERIOR BRASILEIRAS
}

\author{
Terezinha Ribeiro Alvim e João Carlos de Andrade* \\ Instituto de Química, Universidade Estadual de Campinas, CP 6154, 13084-971 Campinas-SP
}

Recebido em 24/11/04; aceito em 13/5/05; publicado na web em 25/11/05

\begin{abstract}
THE IMPORTANCE OF QUALITATIVE ANALYTICAL CHEMISTRY IN CHEMISTRY COURSES IN BRAZILIAN UNIVERSITIES. The results of a survey of institutions offering undergraduate studies, with the objective of evaluating the importance of Qualitative Analytical Chemistry for Chemistry courses in Brazil, are presented and discussed. Judging by the data, the content of the course of Qualitative Analytical Chemistry is considered by the Brazilian institutions offering undergraduate studies to be a body of knowledge essential for the formation of the chemist. This aspect is deemed valid for both baccalaureate and teaching license studies.
\end{abstract}

Keywords: Chemical education; Qualitative Analytical Chemistry; Brazilian institutions.

\section{INTRODUÇÃO}

A disciplina Química Analítica Qualitativa dos cursos de Química tem geralmente como conteúdo programático a teoria do equilíbrio químico em solução aquosa e a análise química qualitativa inorgânica em amostras sólidas ou líquidas.

Os conhecimentos ensinados na parte experimental desta disciplina têm sua origem na Antiguidade. Há cerca de 2000 anos atrás, Caius Plinius Secundus (23-79 d.C.) fez o primeiro registro de um teste químico de análise qualitativa ${ }^{1}$. Esse teste, que visava detectar a contaminação de sulfato de ferro(II) em acetato de cobre(II), consistia em tratar uma tira de papiro embebida em extrato de noz de galha (ácido tânico) com a solução sob exame. Se a tira adquirisse a cor preta, indicava presença do sulfato de ferro(II).

Na segunda metade do século XVII, Robert Boyle (1627- 1691) muito contribuiu para o desenvolvimento da análise química estudando o uso de reações químicas para identificar várias substâncias e introduzindo novos reagentes analíticos, sendo pioneiro no uso do "volatile sulphureous spirit" (sulfeto de hidrogênio) com fins analíticos ${ }^{2}$. Até essa época os métodos de análise por via úmida eram principalmente qualitativos e baseados nas propriedades químicas dos elementos.

Outros pesquisadores, como Otto Tachenius (início do século XVII) e Sigismund Andreas Marggraf (1709 - 1782), também contribuíram para que grande parte das reações químicas conhecidas e usadas em análise qualitativa inorgânica já tivesse sido descoberta até o fim do século XVIII ${ }^{2,3}$. Entretanto, somente no século XIX, a análise sistemática dos elementos foi introduzida através dos trabalhos de Heinrich Rose (1795-1864) e de Carl Remigius Fresenius (1818-1897)2, publicados em 1829 e 1841, respectivamente.

A análise qualitativa apresentou avanços significativos no final do século XIX e no início do século XX, respectivamente, com os trabalhos de Theodor Heinrich Behrens (1843-1905) sobre técnicas microscópicas de análise e de Fritz Feigl (1891-1971) e colaboradores, sobre os testes de toque ("spot tests") ${ }^{1,2,4}$. Estes trabalhos, publicados em livros ${ }^{5,6}$, vieram consolidar essa nova área da análise química.

Através de consulta ao Chemical Abstracts, verificou-se que grande parte dos trabalhos publicados sobre análises qualitativas

*e-mail: dandrade@iqm.unicamp.br até cerca da metade do século XX compreendiam, principalmente, estudos e aplicações de novos reagentes (geralmente orgânicos), novos testes para detecção de cátions e ânions usando técnicas microanalíticas ${ }^{7-30}$, e vários estudos sobre métodos de separação de íons em uma análise sistemática ${ }^{31-50}$. A partir dos anos 60, as publicações sobre Química Analítica Qualitativa tiveram seu foco voltado para as atividades de ensino ${ }^{51-78}$.

Essa situação repercutiu nos currículos dos cursos universitários dos Estados Unidos. Pesquisas realizadas em 1940 ${ }^{79}, 1950^{80}, 1963^{81} \mathrm{e}$ $1966^{82}$ revelaram que, ao longo desses anos, a disciplina de Química Analítica Qualitativa Inorgânica foi desaparecendo dos currículos das instituições de ensino superior americanas, que passaram a incluir parte das suas atividades de laboratório na disciplina de Química Geral. Nesses casos, houve uma redução da carga horária destinada às atividades experimentais e a supressão da parte teórica específica da análise qualitativa, especialmente os equilíbrios químicos.

Vários artigos e opiniões foram publicados sobre esse assunto ${ }^{83-91}$, citando como principal motivo para supressão da disciplina de Qualitativa o fato de que os processos analíticos ali ensinados não tinham valor prático real. Diante disso, Strong $^{89}$ sugeriu que um novo curso de Química Analítica Qualitativa deveria ensinar, na medida do possível, os princípios básicos das técnicas instrumentais, utilizando alguma atividade prática para isso. Simultaneamente, Freiser ${ }^{90}$ propôs uma disciplina na qual a ênfase seria dada aos métodos de separação e não à detecção dos íons.

Outros pesquisadores e educadores de renome, por outro lado, não se mostraram favoráveis a esse novo enfoque. Quando entrevistado por Rudy M. Baum ${ }^{2}$, Henry Taube, Prêmio Nobel de Química de 1983, disse que foi um erro o desaparecimento da análise qualitativa dos currículos das universidades norte-americanas, porque esta constituía um meio de introduzir a química descritiva e de motivar os alunos a estudarem as reações químicas que, em sua opinião, "são o coração da química".

Outro atributo importante do curso de Química Analítica Qualitativa, mencionado por alguns autores ${ }^{77,87,88,93}$, seria o de desenvolver no aluno uma atitude de pesquisador, ao trabalhar com amostras "desconhecidas". Segundo Benedetti-Pichler et al. ${ }^{87}$ o processo de descobrir a composição química de uma amostra requer uma abordagem semelhante à usada em pesquisa. Frank ${ }^{88}$, dentro da mesma idéia, afirmou que a sequiência "pensar e agir" envolvida é 
muitíssimo similar à que se espera de um futuro pesquisador. Senise $^{93}$, ao enfocar o papel da Química Analítica na formação do Químico, destaca a função pedagógica desempenhada pela Química Analítica Qualitativa de estimular o estudante a pensar e a raciocinar criativamente.

Apesar da polêmica, com algumas restrições, havia uma espécie de consenso em torno da idéia de que a análise qualitativa era a melhor forma de ilustrar os princípios do equilíbrio químico em soluções aquosas e a química inorgânica descritiva.

Mesmo com todo esse debate, o fato é que a situação atual da Qualitativa nas instituições de ensino superior brasileiras não é publicamente conhecida. Convive-se com opiniões a favor e contra a manutenção dessa disciplina nos currículos, mas nenhuma posição sobre o assunto é claramente assumida por qualquer grupo de pesquisadores ou professores de Química.

Em vista disso, foi realizada uma pesquisa no $1^{\circ}$ semestre de 2004, na qual foram consultadas as instituições de ensino superior que oferecem Licenciatura e/ou Bacharelado em Química, incluindo todas as regiões geográficas do Brasil, com o objetivo de mostrar e analisar a atual situação da disciplina Química Analítica Qualitativa (ou de seu conteúdo) nos cursos de Química brasileiros.

\section{A PESQUISA}

Após consulta na página do Ministério da Educação ${ }^{94}$ e no Guia do Estudante-Vestibular $2004^{95}$, acessaram-se pela internet as páginas das Instituições de Ensino Superior (IES) que oferecem cursos de licenciatura e bacharelado em Química. Através dessa pesquisa e de contatos diretos com as Coordenações dos Cursos buscou-se saber quais eram as grades curriculares dos mesmos, as suas cargas horárias e os pré-requisitos das disciplinas curriculares, bem como as ementas ou programas das disciplinas da área de Química Analítica. As informações obtidas foram traduzidas em respostas às seguintes perguntas:

$\left.1^{a}\right)$ Em quantos cursos existe uma disciplina com o nome de "Química Analítica Qualitativa” ou similar (ex.: Análise Química Qualitativa, Laboratório de Análise Qualitativa, etc.) na grade curricular?

$2^{a}$ ) Em quantos cursos o conteúdo "análise qualitativa de cátions e ânions por métodos químicos" faz parte do programa de uma disciplina da área de Química Analítica?

$3^{\text {a }) ~ E m ~ q u a n t o s ~ c u r s o s ~ o ~ c o n t e u ́ d o ~ " a n a ́ l i s e ~ q u a l i t a t i v a ~ d e ~ c a ́ t i o n s ~ e ~}$ ânions por métodos químicos" constitui todo o programa prático e em quantos ele é apenas uma parte do programa de uma disciplina da área de Química Analítica?

$4^{\mathrm{a}}$ ) Qual é a carga horária atribuída à disciplina contendo o conteúdo mencionado acima?

$\left.5^{\mathrm{a}}\right)$ Em que momento dos cursos (período, etapa, semestre, ano, etc.) a disciplina é oferecida e qual(is) é(são) o(s) pré-requisito(s) para a mesma?

$6^{\text {a }) ~ E m ~ q u a n t o s ~ c u r s o s ~ e s s e ~ c o n t e u ́ d o ~ e ́ ~ e n s i n a d o ~ e m ~ d i s c i p l i n a s ~ d e ~}$ Química Geral ou Inorgânica?

Das 117 IES brasileiras contatadas, 66 forneceram todas as informações solicitadas, incluindo instituições públicas e particulares (Tabela 1).

\section{RESULTADOS E DISCUSSÃO}

As respostas às três primeiras perguntas (Figuras 1-3) mostram que as IES brasileiras apresentam um enfoque bastante distinto das instituições americanas, em relação ao ensino da análise qualitativa inorgânica. Fica bem claro que no Brasil esse conjunto de conhecimentos ainda é tido como relevante dentro do ensino da Química e merecedor de uma disciplina específica de Química Analítica para ministrá-lo. Os dados colhidos parecem indicar que essa conduta é uniforme e não é influenciada nem pela natureza da instituição (pública ou particular) nem pela região geográfica do país. Cinqüenta dos sessenta e seis cursos analisados têm a análise de cátions e ânions como todo o programa da parte experimental de uma disciplina de Química Analítica, enquanto que em 12 cursos esse conteúdo é um tópico do programa da disciplina que também contém tópicos da análise quantitativa, como gravimetria e volumetria (Figura 3).

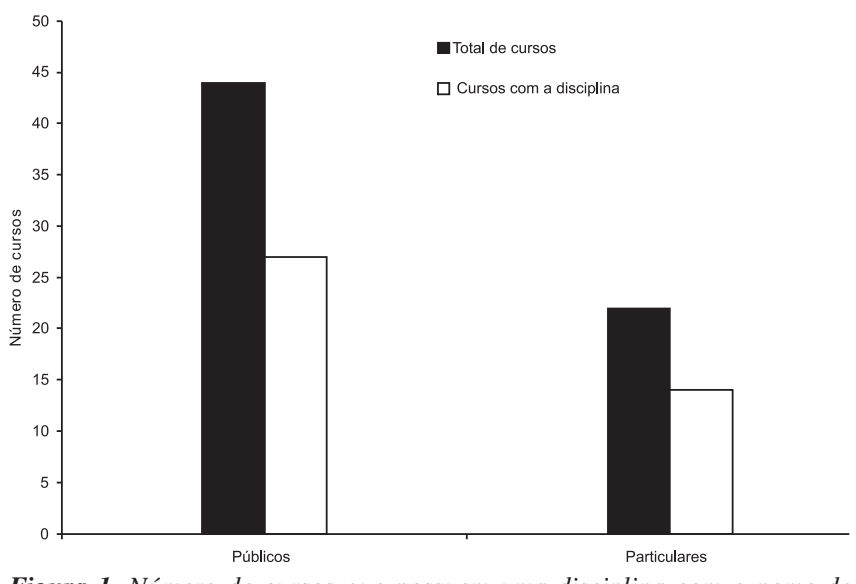

Figura 1. Número de cursos que possuem uma disciplina com o nome de "Química Analítica Qualitativa" (ou similar) em suas grades curriculares

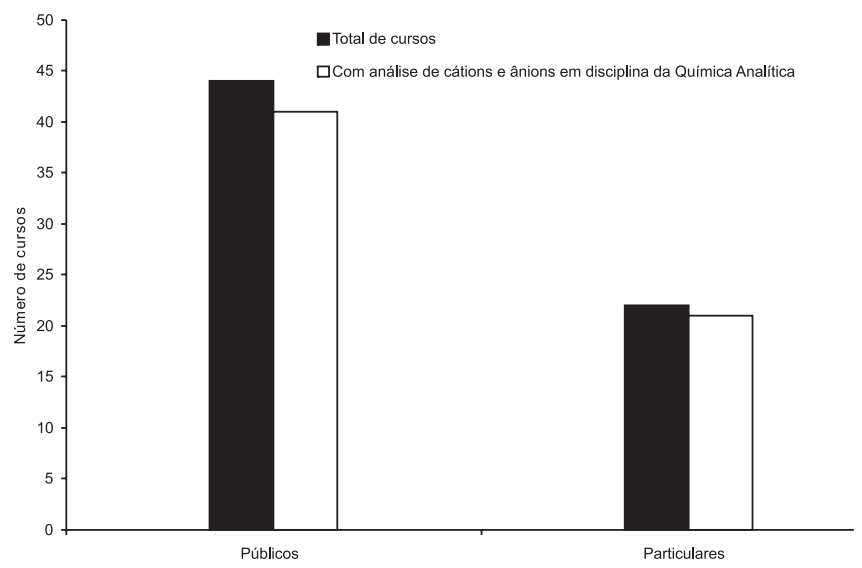

Figura 2. Número de cursos que possuem uma disciplina da área de Química Analítica contendo "análise química qualitativa inorgânica (teoria e prática)" em seu programa

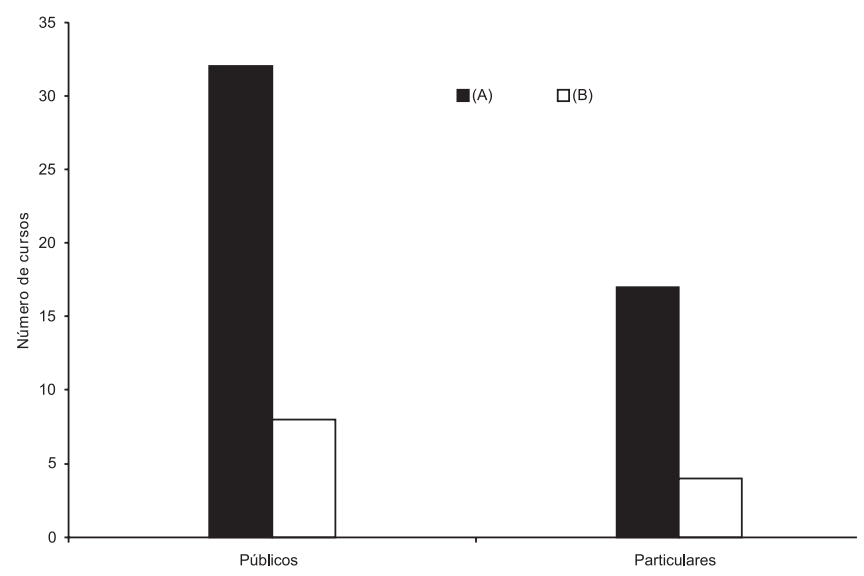

Figura 3. Número de cursos que apresenta o conteúdo "análise qualitativa de cátions e ânions por métodos químicos" no programa da parte experimental da disciplina. (A) como programa completo; (B) apenas como item do programa 
Tabela 1. Instituições de Ensino Superior que forneceram as informações solicitadas e seus respectivos cursos

\begin{tabular}{|c|c|c|}
\hline INSTITUIÇÃO & CURSO(S)* & NATUREZA JURÍDICA** \\
\hline Centro Federal de Educação Tecnológica de Goiás & QI & Pública \\
\hline Faculdade de Filosofia Ciências e Letras de Ribeirão Preto/USP & $\mathrm{B}, \mathrm{L}$ e QT & Pública \\
\hline Instituto de Química de São Carlos/USP & $\mathrm{B}$ & Pública \\
\hline Universidade de São Paulo & $\mathrm{B}, \mathrm{L}$ e QT & Pública \\
\hline Universidade do Estado do Rio de Janeiro & $\mathrm{L}$ & Pública \\
\hline Universidade Estadual da Paraíba & $\mathrm{B}, \mathrm{L}$ e QT & Pública \\
\hline Universidade Estadual de Campinas & $\mathrm{B}, \mathrm{L}$ e QT & Pública \\
\hline Universidade Estadual de Goiás & QI & Pública \\
\hline Universidade Estadual de Londrina & $\mathrm{B}, \mathrm{L}$ e QT & Pública \\
\hline Universidade Estadual de Maringá & B e L & Pública \\
\hline Universidade Estadual de Santa Cruz & $\mathrm{L}$ & Pública \\
\hline Universidade Estadual do Ceará & $\mathrm{L}$ & Pública \\
\hline Universidade Estadual do Centro-Oeste & $\mathrm{B}, \mathrm{L}$ e QT & Pública \\
\hline Universidade Estadual do Mato Grosso do Sul & $\mathrm{L}$ & Pública \\
\hline Universidade Estadual do Oeste do Paraná & $\mathrm{B}, \mathrm{L}$ e QT & Pública \\
\hline Universidade Estadual do Piauí & $\mathrm{L}$ & Pública \\
\hline Universidade Estadual do Rio Grande do Sul & QA & Pública \\
\hline Universidade Estadual do Sudoeste da Bahia & $\mathrm{B}, \mathrm{L}$ e QT & Pública \\
\hline Universidade Estadual Paulista & $\mathrm{B}, \mathrm{L}$ e QT & Pública \\
\hline Universidade Federal de Santa Maria & L e QI & Pública \\
\hline Universidade Federal da Paraíba & B e L & Pública \\
\hline Universidade Federal de Goiás & $\mathrm{B}, \mathrm{L}$ e QI & Pública \\
\hline Universidade Federal de Juiz de Fora & B e L & Pública \\
\hline Universidade Federal de Minas Gerais & B e L & Pública \\
\hline Universidade Federal de Pernambuco & $\mathrm{B}, \mathrm{L}$ e QI & Pública \\
\hline Universidade Federal de Santa Catarina & $\mathrm{B}, \mathrm{L}$ e QT & Pública \\
\hline Universidade Federal do Espírito Santo & $\mathrm{L}$ & Pública \\
\hline Universidade Federal do Pará & $\mathrm{B}, \mathrm{L}$ e QI & Pública \\
\hline Universidade Federal do Paraná & B e L & Pública \\
\hline Universidade Federal do Rio de Janeiro & $\mathrm{B}$ e L & Pública \\
\hline Universidade Federal do Rio Grande do Sul & B e L & Pública \\
\hline Universidade Federal Fluminense & $\mathrm{B}, \mathrm{L}$ e QI & Pública \\
\hline Universidade Federal Rural do Rio de Janeiro & B e L & Pública \\
\hline Fundação Universidade de Brasília & B e L & Mista \\
\hline Fundação Universidade Federal de Mato Grosso & B e L & Mista \\
\hline Fundação Universidade Federal de Ouro Preto & QI & Mista \\
\hline Fundação Universidade Federal de Pelotas & $\mathrm{B}, \mathrm{L}$ e QA & Mista \\
\hline Fundação Universidade Federal de São Carlos & B e L & Mista \\
\hline Fundação Universidade Federal de São João Del Rei & $\mathrm{L}$ & Mista \\
\hline Fundação Universidade Federal de Sergipe & $\mathrm{B}, \mathrm{L}$ e QI & Mista \\
\hline Fundação Universidade Federal de Uberlândia & B e L & Mista \\
\hline Fundação Universidade Federal de Viçosa & $\mathrm{B}$ e L & Mista \\
\hline Fundação Universidade Federal do Piauí & $\mathrm{B}$ e $\mathrm{L}$ & Mista \\
\hline Fundação Universidade Federal do Rio Grande ${ }^{\#}$ & $\mathrm{~L}$ & Mista \\
\hline Centro Universitário La Salle & L e QT & Particular \\
\hline Centro Universitário Univates & QA & Particular \\
\hline Faculdades Integradas do Vale do Ribeira & $\mathrm{L}$ & Particular \\
\hline Fundação Universidade Regional de Blumenau & $\mathrm{B}$ e $\mathrm{L}$ & Particular \\
\hline Instituto Manchester Paulista de Ensino Superior & B & Particular \\
\hline Instituto Municipal de Ensino Superior de Assis & QA & Particular \\
\hline Pontifícia Universidade Católica do Rio de Janeiro & $\mathrm{B}, \mathrm{L}$ e QI & Particular \\
\hline Pontifícia Universidade Católica do Rio Grande do Sul & L e QI & Particular \\
\hline Universidade Católica de Brasília & $\mathrm{L}$ & Particular \\
\hline Universidade Católica de Pelotas & QAm & Particular \\
\hline Universidade Católica de Pernambuco & $\mathrm{B}, \mathrm{L}$ e $\mathrm{QI}$ & Particular \\
\hline Universidade da Região de Joinville & QI & Particular \\
\hline Universidade de Passo Fundo & $\mathrm{B}$ e L & Particular \\
\hline Universidade de Santa Cruz do Sul & L e QI & Particular \\
\hline Universidade do Contestado & QA & Particular \\
\hline Universidade do Sul de Santa Catarina & $\mathrm{L}$ & Particular \\
\hline
\end{tabular}


Tabela 1. (cont.)

\begin{tabular}{lcc}
\hline \multicolumn{1}{c}{ INSTITUIÇÃO } & CURSO(S)* & NATUREZA JURÍDICA** \\
\hline Universidade Metodista de Piracicaba & B & Particular \\
Universidade para o Desenvolvimento do Alto Vale do Itajaí & QA & Particular \\
Universidade Presbiteriana Mackenzie & L & Particular \\
Universidade Regional do Noroeste do Estado do Rio Grande do Sul & B, L QA & Particular \\
Universidade Regional Integrada do Alto Uruguai e das Missões & B e L & Particular \\
Universidade São Francisco & L & Particular \\
\hline
\end{tabular}

* B = Bacharelado; L = Licenciatura; QI = Química Industrial; QT = Química Tecnológica; QA = Química de Alimentos ; QAm = Química Ambiental. ** O termo Particular foi usado para designar as instituições de natureza privada, com ou sem fins lucrativos. *Rio Grande do Sul

Para se obter a resposta à quarta pergunta, foi contabilizada a carga horária total despendida com o ensino da teoria dos equilíbrios químicos e dos métodos de separação e identificação dos cátions e ânions, nos 50 cursos. Como as cargas horárias totais apresentaram uma grande variação entre os cursos (60 a 200 h), levantou-se o número de cursos por faixa de carga horária (Figura 4). Os cursos particulares tendem a estabelecer uma carga horária menor quando comparados aos cursos públicos, que se distribuem igualmente em três faixas de carga horária. Deve-se notar que apenas um curso particular dedica uma carga horária em torno de $120 \mathrm{~h}$ para o ensino desses conteúdos. Isto indica que no Brasil os métodos químicos de análise qualitativa inorgânica e seus fundamentos teóricos são considerados conhecimentos necessários aos estudantes, pois os cursos de Química dedicam uma carga horária significativa à essa disciplina, notadamente nos cursos públicos.

Em relação à quinta pergunta, verificou-se que, com exceção de um curso, todos os outros oferecem esse conteúdo a partir do $2^{\circ}$ semestre. Mais exatamente, foi observado que a maioria deles oferece a disciplina a partir do $3^{\circ}$ semestre (Figura 5). Como a quími-

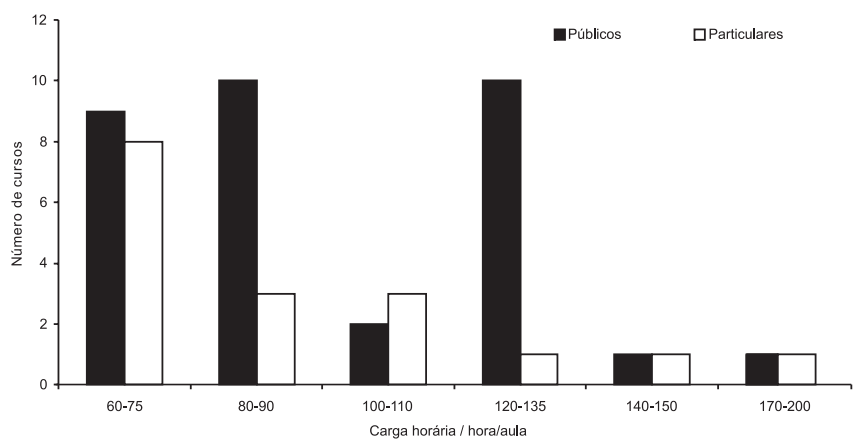

Figura 4. Cargas horárias atribuídas às disciplinas cujos conteúdos programáticos são equilíbrio químico e análise química qualitativa de cátions e ânions (teoria e prática)

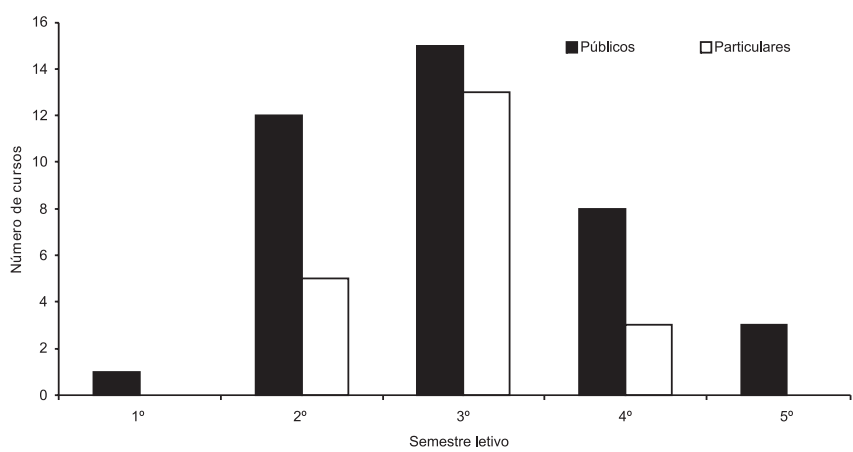

Figura 5. Indicações sobre qual semestre letivo a disciplina com conteúdo programático "análise química qualitativa de cátions e ânions" é oferecida ca analítica qualitativa inorgânica caracteriza-se por um conjunto de conhecimentos técnicos e científicos aplicados, fundamentados em conceitos e teorias da química inorgânica e da físico-química, as IES brasileiras parecem considerar que seja difícil para um aluno ingressante executar os experimentos da análise qualitativa, sem um conhecimento prévio dos aspectos teóricos da matéria e um treinamento básico em técnicas de laboratório.

Quanto aos pré-requisitos, apenas 34 cursos mencionam esta exigência de forma explícita. Destes, 26 estabeleceram como pré-requisito apenas a disciplina de Química Geral. Entre os restantes, 3 mencionam apenas a disciplina de Química Inorgânica, 1 menciona apenas uma disciplina da Química Analítica e 3 as disciplinas de Química Geral e Química Inorgânica, conjuntamente.

Além disso, pode-se destacar que apenas 2 cursos ministram esse conteúdo em alguma disciplina do ciclo básico (Química Geral ou simplesmente Química). Também foi constatado que 2 cursos públicos não têm esse conteúdo em seus currículos obrigatórios.

\section{CONCLUSÃO}

Pelos dados analisados, o conteúdo da disciplina Química Analítica Qualitativa (ou equivalente) parece ser considerado pelas IES brasileiras como um conjunto de conhecimentos essencial e obrigatório para a formação do Químico. Isto é válido tanto para os cursos de licenciatura como para os de bacharelado, em suas diversas modalidades, indicando que qualquer mudança significativa no quadro atual deve ser discutida em profundidade.

De fato, a importância da disciplina de Química Analítica Qualitativa fica evidente quando se mostra que várias reações empregadas nas práticas de laboratório são fundamentos para vários métodos quantitativos de referência ${ }^{96-98}$, empregados em diversos laboratórios. Além disso, diversos testes qualitativos são empregados rotineiramente no controle de qualidade de fármacos ${ }^{97} \mathrm{e}$ outros materiais ${ }^{98}$.

Isso mostra a limitação dos argumentos dos docentes americanos e que as IES brasileiras, públicas e privadas, incluindo as de grande notoriedade, reafirmam a importância da disciplina Química Analítica Qualitativa dentro da realidade acadêmica brasileira. É provável que os estudantes venham a ter maior interesse pela matéria se os docentes mostrarem os inúmeros usos práticos e reais dos conhecimentos contidos nas ementas dessa disciplina.

\section{AGRADECIMENTOS}

A todas as instituições que contribuíram com essa pesquisa fornecendo-nos os dados solicitados.

\section{REFERÊNCIAS}

1. Hillis, M. O.; J. Chem. Educ. 1945, 22, 348. 
2. Szabadváry, F.; History of Analytical Chemistry, Pergamon Press Ltd.: Londres, 1966.

3. Welcher, F. J.; J. Chem. Educ. 1957, 34, 389.

4. van Nieuwenburg, C. J.; Analyst 1956, 81, 450

5. Feigl, F.; Anger, V.; Spot Tests in Inorganic Analysis, $6^{\text {th }}$ ed., Elsevier Publishing Company: Amsterdam, 1972.

6. Feigl, F.; Anger, V.; Spot Tests in Organic Analysis, $7^{\text {th }}$ ed., Elsevier Publishing Company: Amsterdam, 1966.

7. Feigl, F.; Z. Anal. Chem. 1918, 57, 135 (CA 12: 2173).

8. Feigl, F.; Stern, R.; Z. Anal. Chem. 1921, 60, 1 (CA 15: 2598).

9. Feigl, F. ; Mikrochemie 1923, 1, 74. (CA 18: 1624).

10. Feigl, F. ; Ber. 1924, 57B , 759 (CA 18: 2663).

11. Denigès, G.; Bull. Soc. Pharm. Bordeaux 1924, 62, 65 (CA 18: 2298).

12. Feigl, F. ; Mikrochemie 1924, 2, 186 (CA 19: 1108).

13. Fischer, H.; Mikrochemie 1930, 2,319 (CA 25: 893).

14. Feigl, F.; Krumholz, P.; Rajmann, E.; Mikrochemie 1931, 3, 395 (CA 25: 4197).

15. Fiegl, F.; Hamburg, H.; Z. Anal. Chem. 1931, 86, 1 (CA 25: 5866).

16. Grünsteidl, E.; Mikrochemie 1932, 12,169 (CA 27: 678).

17. Feigl, F. ; Mikrochemie 1933, 13, 129 (CA 27: 3895).

18. Fischer, H.; Angew. Chem. 1934, 47, 685 (CA 29: 69').

19. Benedetti-Pichler, A. A.; Spikes, W. F.; Mikrochemie 1934, 15, 271 (CA 29: $\left.69^{6}\right)$.

20. Augusti, S.; Mikrochemie 1935, 17, 113 (CA 29: 53847).

21. van Nieuwenburg, C. J.; van der Hoek, T.; Mikrochemie 1935, 18, 175 (CA 30: $\left.43^{3}\right)$.

22. Tananaev, N. A.; Budkevich, A. A.; Z. Anal. Chem. 1935, 103, 353 (CA 30: $1328^{2}$.

23. Tananaev, N. A.; Shulepova, V. A.; J. Appl. Chem.(U.S.S.R.), 1935, 8, 1070 (CA 30: $5149^{1}$ ).

24. Rossi, L. ; Quim. Ind. 1935, 12, 277 (CA 30: 21315).

25. Pearson, J. G.; Chem. Eng. Mining. Rev. 1936, 28, 108 (CA 30: 25174).

26. Yanowski, L. K.; Hynes, W. A.; Mikrochemie 1937, 23, 143 (CA 32: 16113).

27. Martini, A. ; Pub. Inst. Invest. Microquim. 1940, 4, 25 (CA 36: 366 $\left.{ }^{8}\right)$.

28. Martini, A.; Graf, J. C. B. ; Pub. Inst. Invest. Microquim. 1939, 3, 55 (CA 36: $\left.6434^{3}\right)$.

29. Manolov, K. R.; Mikrochim. Acta 1962, 977 (CA 57: 14416h).

30. Grienpink, B. F. A.; Veldman, J.; Mikrochim. Ichnoanal. Acta 1964, 5, 750 (CA 61: 12607h).

31. Noyes, A. A.; J. Am. Chem. Soc.1912, 34, 609.

32. Welch, J. M.; Weber, H. C. P.; J. Am. Chem. Soc. 1916, 38, 1011.

33. Weber, H. C. P.; Winkelmann, H. A.; J. Am. Chem. Soc. 1916, 38, 2000.

34. Welcher, F. J.; Briscoe, H. T.; Chem. News 1932, 145, 161 (CA 27: 44).

35. Scheinkmann, A. I.; Z. Anal. Chem. 1933, 9, 415 (CA 27: 1841).

36. Smith, T. B.; J. Chem. Soc. 1933, 253.

37. Sconzo, A.; Ann. Chim. Appl. 1933, 23, 215 (CA 27: 5029).

38. Macri, V.; Boll. Chim. Farm. 1935, 74, 345 (CA 29: 7213').

39. Puzevskaya, S. M.; J. Gen. Chem. (U.S.S.R.) 1935, 5, 498 (CA 29: 72249).

40. Benedetti-Pichler, A. A.; Rachele, J. R.; Ind. Eng. Chem., Anal. Ed. 1937, 9, 589.

41. Benedetti-Pichler, A. A.; Rachele, J. R.; Mikrochemie 1938, 24, 16 (CA 32: $\left.4469^{7}\right)$.

42. Austin, G. J.; Analyst 1947, 72, 443.

43. Taimni, I. K.; Agarwal, R. P.; Anal. Chim. Acta 1953, 9, 208.

44. Swift, E. H.; Niemann, C.; Anal. Chem. 1954, 26, 538.

45. Vogel, A. I.; Cresswell, W. T.; Jeffery, G. H.; Leicester, J.; Analyst 1956, $81,244$.

46. Taimni, I. K.; Lal, M.; Anal. Chim. Acta 1957, 17, 367.
47. Salaria, G. B. S.; Anal. Chim. Acta 1959, $21,312$.

48. Henry, A. J.; Analyst 1964, 89, 255

49. Poonia, N. S.; Bhagwat, V. W.; Mikrochim. Acta 1969, 5, 1008

50. Lumme, P.;Tummavuori, J.; Acta Chem. Scand. 1973, 27, 851.

51. Lehrman,L.; Dorenbush, M.; Meisler, N.; J. Chem. Educ. 1960, 37, 407.

52. Hahn, R. B.; Sanders, C. H.; J. Chem. Educ. 1960, 37, 408.

53. Hahn, R. B.; Sanders, C. H.; Gutnikov, G.; J. Chem. Educ. 1960, 37, 412.

54. Armstrong, A. R.; J. Chem. Educ. 1960, 37, 413

55. Chandra, R.; J. Chem. Educ. 1961, 38, 409.

56. Vavoulis, A.; J. Chem. Educ. 1962, 39, 395.

57. Paul, A. D.; Gibson,Jr., J. A.; J. Chem. Educ. 1962, 39, 398.

58. Whitehead, T. H.; Hatcher, G. K.; J. Chem. Educ. 1962, 39, 399.

59. Rich, R.; J. Chem. Educ. 1962, 39, 400.

60. O'Donnell, T. A.; J. Chem. Educ. 1963, 40, 415.

61. Paul, A. D.; Gibson,Jr., J. A.; J. Chem. Educ. 1963, 40, 417.

62. Poonia, N. S.; Gupta, H. K. L.; J. Chem. Educ. 1964, 41, 439.

63. Paul, A. D.; Gibson,Jr., J. A.; J. Chem. Educ. 1965, 42, 440.

64. Packer, J. E.; J. Chem. Educ. 1966, 44, 480.

65. Poonia, N. S.; Gupta, H. K. L.; J. Chem. Educ. 1967, 44, 480.

66. Thompson, M.; Bixler, J. W.; J. Chem. Educ. 1971, 48, 113.

67. Ophadt, C. E.; J. Chem. Educ. 1974, 51, 415.

68. Haendler, B. L.; Cook, R. ; Siemiencow, G.; J. Chem. Educ. 1982, 59, 333.

69. Cole, G. M.; Waggoner, W. H.; J. Chem. Educ. 1983, 60, 135.

70. Kistner, C. R.; Robinson, P. J.; J. Chem. Educ. 1983, 60, 1071

71. Kilner, C.; J. Chem. Educ. 1985, 62, 80.

72. Vitale, D.; J. Chem. Educ. 1990, 67, 64.

73. Blankenship, J. F.; Costello, S.; Sprouse, M.; Settle, Jr., F. A.; Bolen, R. H.; Pleva, M. A.; J. Chem. Educ. 1991, 68, A65.

74. Solomon, S.; Fülep-Poszmik, A.; Lee, A.; J. Chem. Educ. 1991, 68, 328.

75. Petty, J. T.; J. Chem. Educ. 1991, 68, 942

76. Laing, M.; J. Chem. Educ. 1993, 70, 666.

77. Tan, Y. S. S.; Tan, B. H. I.; Lee, H. K.; Yan, Y. K.; Hor, T. S. A.; J. Chem. Educ. 1998, 75, 456.

78. Chakraborty, A.; Chakraborty, A.; Chakravarti, A. K.; J. Indian Chem. Soc. 2002, 79,197.

79. Reed, R. D.; Cortelyou, W. P.; Calandra, A.; J. Chem. Educ. 1940, 17, 220.

80. Bacon, E. K.; Heath, F. H.; Martin, C. S.; Reed, R. D.; Rogers, H. W.; Weidner, B. V.; J. Chem. Educ. 1950, 27, 675.

81. Hovey, N. W.; J. Chem. Educ. 1963, 40, 410.

82. Krohn, A.; J. Chem. Educ. 1966, 43, 419.

83. Sears, G. W.; J. Chem. Educ. 1927, 4, 627.

84. Guy, J. S.; J. Chem. Educ. 1928, 5, 573.

85. Furman, N. H.; J. Chem. Educ. 1928, 5, 946

86. Wasley, W. L.; J. Chem. Educ. 1946, 23, 357.

87. Benedetti-Pichler, A. A.; Schneider, F.; Steinbach, O. F.; J. Chem. Educ. 1957, 34, 381

88. Frank, R. E.; J. Chem. Educ. 1957, 34, 383.

89. Strong III, F. C.; J. Chem. Educ. 1957, 34, 400.

90. Freiser, H.; J. Chem. Educ. 1957, 34, 387.

91. Stubbs, M. F.; J. Chem. Educ. 1959, 36, 387.

92. Baum, R. M.; Chem. Eng. News 1984, 62, 31.

93. Senise, P. E. A.; Quim. Nova 1982, 5, 137.

94. http://www.mec.gov.br/sesu/ies.shtm, acessada em Janeiro 2004.

95. Guia do Estudante Vestibular, ed. 2004, Ed. Abril S. A.: São Paulo.

96. de Andrade, J. C.; Alvim, T. R.; Resumos do $43^{\circ}$ Congresso Brasileiro de Química da Associação Brasileira de Química, Ouro Preto, Brasil, 2003.

97. Farmacopéia brasileira, $4^{\mathrm{a}}$ ed., São Paulo: Atheneu, 1988.

98. ASTM Standards Source CD 2003; West Conshohocken PA. 